\title{
An Expeditious Synthesis of 6-Amido-(1H,3H)-Pyrimidine- 2,4-Diones from Uracil-6-Carboxylic Acid
}

\author{
Md. Wahab Khan, Md. Enamul Haque and Md. Faiaz Ahmed \\ Department of Chemistry, Bangladesh University of Engineering \& Technology (BUET), \\ Dhaka-1000, Bangladesh
}

Received: October 31, 2013; Accepted: November 05, 2013; Published (web): June 29, 2014

\begin{abstract}
Dichloro pyrimidine-6-carbonylchloride (2) was synthesized by refluxing uracil-6-carboxylic acid (orotic acid) with phosphorus oxychloride and phosphorus pentachloride. Compound (2) underwent a smooth coupling reaction with a number of substituted arylamines to yield 2, 4-dichloro-6-amidopyrimidines (8-12) which were converted to the corresponding 2, 4-dimethoxy-6-amidopyrimidines (13-17) on treatment with sodium methoxide in methanol. Compounds 13-17 afforded 6-amido-( $1 H, 3 H)$-pyrimidine-2, 4-diones (18-22) in good yield on refluxing with $6 \mathrm{M}$ hydrochloric acid. These pyrimidinone derivatives may exhibit antiviral actitities.
\end{abstract}

Key word: Pyrimidine, uracil, orotic acid, phosphorus oxychloride, arylamines, antiviral

\section{INTRODUCTION}

The importance of 5-substituted derivatives of uracil as anticancer and antiviral agents is wellestablished. 5-Fluorouracil ${ }^{1}$ (5-FU) $\mathbf{I}$ and the corresponding 2-deoxyribonucleoside (FUdR) are being used as anticancer agents whereas 5-iodo-2deoxyuridine $^{2}$ is of importance as an antiviral agent. Dihydroalkoxybenzyloxopyrimidines (DABOs) II are a new class of specific inhibitors of human immunodeficiency virus type 1 (HIV-1) which possess a benzyl moiety and an alkyl (cycloalkyl) chain linked through an oxygen bridge to the uracil or

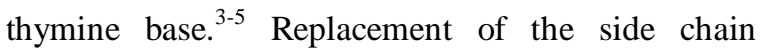
oxygen with sulfur atom furnished thio-DABOs, which showed increased anti-HIV activity. ${ }^{6}$ Miyasaka et al have reported that 1-[(2-hydroxyethoxy) methyl]-6-phenylthio) thymine (HEPT) III has potent and selective in vitro activity against HIV $-1{ }^{7}$

Correspondence to: Md. Wahab Khan

E mail: mwkhan@chem.buet.ac.bd

Dhaka Univ. J. Pharm. Sci. 13(1): 27-29, 2014 (June)<smiles>O=c1[nH]c(=O)n(I)cc1F</smiles><smiles>CCOc1nc(Cc2ccccc2)c(P)c(=O)[nH]1</smiles>

II<smiles>Cc1c(Sc2ccccc2)n(COCO)c(=O)[nH]c1=O</smiles>

The synthesis and antviral activities of a series of 6-arylmethyl-1-allyloxymethyl)-5-alkyluracil derivatives have been reported. ${ }^{8}$ Recently, we have reported a synthesis of 4-acyl-2, 6-dioxo-1, 2, 3, 6tetrahydropyrimidines (6-acyluracils and 4-acyl-6aryl-2-oxo-2, 3-dihydropyrimidines. ${ }^{9}$

In view of the significant biological activities of various 6-substituted uracils and related pyrimidine derivatives we became interested in developing methods for the synthesis of novel 6-substituted 
pyrimidines. In this paper we report a very facile method for the synthesis of a number of 6-amidopyrimidine-2,4-diones from orotic acid.

\section{MATERIALS AND METHODS}

Melting points were determined in open capillary tubes on Gallenkamp (England) melting point apparatus and were uncorrected. IR spectra were recorded on a Shimadzu FTIR spectrophotometer and UV spectra were recorded in dry EtOH with a Shimadzu visible spectrophotometer. ${ }^{1} \mathrm{H}$ NMR and ${ }^{13} \mathrm{C}$ NMR spectra were recorded on a Bruker DPX 400 spectrophotometer $(400 \mathrm{MHz})$ using tetramethylsilane as internal reference. Analytical thin-layer chromatography (TLC) was performed on precoated silica gel $60 \mathrm{~F}_{254}$ (E. Merck), and the spots were visualized with UV light. Column chromatography was performed on silica gel (60-120 mesh). Elemental analyses $(\mathrm{C}, \mathrm{H}, \mathrm{N})$ were carried out on a Perkin- Elmer $240 \mathrm{C}$ analyser. Orotic acid, $\mathrm{POCl}_{3}$, primary arylamine, and other reagents were purchased from E. Merck (Germany) and Fluka (Switzerland).

Preparation of 2,4-dichloropyrimidine-6carbonyl chloride (2). A mixture of 2,4-dioxo-1,3, 5-trihydro pyrimidine-6-carboxylic acid (Orotic acid) $5.0 \mathrm{~g}(0.032 \mathrm{~mol})$ and phosphorus oxychloride $\left(\mathrm{POCl}_{3}, 40 \mathrm{ml}\right)$ was refluxed for 24 hours at 105-108 ${ }^{\circ} \mathrm{C}$, then phosphorus pentachloride $(15 \mathrm{~g}, 0.072 \mathrm{~mol})$ was added into the reaction mixture. The mixture was again refluxed for 24 hours. Phosphorus oxychloride was removed under reduced pressure. The residue was distilled under reduced pressure and 2,4dichloropyrimidine-6-carbonyl chloride (2) (4.0 g) was obtained as dense colorless liquid. An analytical sample was prepared by redistillation of the product, b.p.108-109 ${ }^{\circ} \mathrm{C}(5.0 \mathrm{~mm})\left[\mathrm{Lit}^{17} 109{ }^{\circ} \mathrm{C}(5.0 \mathrm{~mm})\right]$. Anal. Calcd. For $\mathrm{C}_{5} \mathrm{HN}_{2} \mathrm{OCl}_{3}$ : 28.40; N, 13.24; $\mathrm{Cl}$, 50.43. Found: C, 28.25; N, 13.35; Cl, 50.23.

Synthesis of 2,4-dichloro-6-substituted phenylamido pyrimidines (8-12). The substituted anilines 3-7 were dissolved in benzene and added to the cold solution of 2,4-dichloropyrimidine-6-carbonyl chloride $(5.0 \mathrm{~g}, 23.64 \mathrm{mmol})$ drop wise. The mixture was then allowed to warm upto room temperature and stirred at room temperature $\left(25^{\circ} \mathrm{C}\right)$ for 2 hours. The mixture was kept at $0-5^{\circ} \mathrm{C}$ for overnight. Then the mixture was concentrated by evaporation of benzene under reduced pressure, washed with distilled water $(100 \mathrm{ml})$ followed by saturated aqueous solution of sodium hydrogen carbonate $(2 \mathrm{x}$ $50 \mathrm{ml})$ and extracted with chloroform $(3 \times 50 \mathrm{ml})$. The combined organic layer was washed with distilled water $(2 \times 25 \mathrm{ml})$, dried over anhydrous. $\mathrm{NaSO}_{4}$, filtered, and concentrated under reduced pressure. The crude products were purified by column chromatography and crystallized from methanol to afford the desired products (8-12).

2,4-dichloro-6-p-methoxyphenylamido pyrimidine (8). Yellowish amorphous powder, yield: $5.85 \mathrm{~g}$ (82\%), mp 147-149 ${ }^{\circ} \mathrm{C}$; UV (EtOH): $\lambda_{\max }$ $352.00 \mathrm{~nm}$; IR (KBr): $v_{\max } 3354.0,1689.5,1564.2$, $1529.4,1508.2,1415.7,1298.0,1253.6,1250.0$, 1033.8, 829.3 \& $788.8 \mathrm{~cm}^{-1} ;{ }^{1} \mathrm{H}$ NMR $(400 \mathrm{MHz}$, $\left.\mathrm{CDCl}_{3}\right): \delta=3.83\left(3 \mathrm{H}, \mathrm{s}, \mathrm{Ar}-\mathrm{OCH}_{3}\right), 6.94(2 \mathrm{H}, \mathrm{d}, J=$ $9.02 \mathrm{~Hz}, \mathrm{Ar}-\mathrm{H}), 7.65(2 \mathrm{H}, \mathrm{d}, J=9.00, \mathrm{Ar}-\mathrm{H}), 8.17$ $(1 \mathrm{H}, \mathrm{s}, \mathrm{H}-5), 9.42$ (s, -NH-); ${ }^{13} \mathrm{C}$ NMR $100 \mathrm{MHz}$, $\left.\mathrm{CDCl}_{3}\right): \delta=54.50,55.15 \& 55.54\left(-\mathrm{OCH}_{3}\right), 100.39$ (Ur-CH), 114.34, 121.51 (Ar-CH), 130.38, 133.09 (Ar-C), 156.80, 159.22, 159.97 (Ur-C), 165.13 $(\mathrm{C}=\mathrm{O})$. Anal. Calcd for $\mathrm{C}_{12} \mathrm{H}_{9} \mathrm{Cl}_{2} \mathrm{~N}_{3} \mathrm{O}_{2}: \mathrm{C}, 48.34 ; \mathrm{H}$, 3.04; N, 14.09. Found: C, 47.40; H, 3.22; N, 13.65 .

2,4-dichloro-6-p-chlorophenylamido pyrimidine (9). Reddish fine crystal yield $6.07 \mathrm{~g}$, (85\%), mp 166$167^{\circ} \mathrm{C}$; UV (EtOH): $\lambda_{\max } 306.00 \mathrm{~nm}$; IR (KBr): $v_{\max }$ $3359.8,1685.7,1566.1,1527.5,1488.9,1400.2$, $1247.9,837.0, \& 758.0 \mathrm{~cm}^{-1} ;{ }^{1} \mathrm{H}$ NMR $(400 \mathrm{MHz}$, $\left.\mathrm{CDCl}_{3}\right): \delta=7.36(2 \mathrm{H}, \mathrm{d}, J=8.2 \mathrm{~Hz}, \mathrm{Ar}-\mathrm{H}), 7.68(2 \mathrm{H}$, d, $J=8.14 \mathrm{~Hz}, \mathrm{Ar}-\mathrm{H}), 8.14(1 \mathrm{H}, \mathrm{s}, \mathrm{H}-5), 9.49$ (s, $\mathrm{NH}-) ;{ }^{13} \mathrm{C}$ NMR $\left(100 \mathrm{MHz}, \mathrm{CDCl}_{3}\right): \delta=118.16$ (Ur$\mathrm{CH}), \quad 119.01,119.89,124.43,126.57$ (Ar-CH), 130.06, 131.21 (Ar-C), 157.90, 159.19, 160.02 (UrC), $165.41(\mathrm{C}=\mathrm{O})$; Anal. Calcd for $\mathrm{C}_{11} \mathrm{H}_{6} \mathrm{Cl}_{3} \mathrm{~N}_{3} \mathrm{O}$ : C, 43.67; H, 2.00; N, 13.89. Found: C, 43.81; H, 2.15; $\mathrm{N}, 13.85$.

2,4-dichloro-6-m-chlorophenylamido pyrimidine (10). Reddish amorphous powder, yield: $5.93 \mathrm{~g}$ (83\%), mp 160-162 ${ }^{\circ} \mathrm{C}$; UV (EtOH): $\lambda_{\max } 324.00 \mathrm{~nm}$; 
IR (KBr): $v_{\max } \quad 3340.5,1695.3,1593.1,1525.6$, $1251.7 \& 680.8 \mathrm{~cm}^{-1} ;{ }^{1} \mathrm{H}$ NMR $\left(400 \mathrm{MHz}, \mathrm{CDCl}_{3}\right): \delta$ $=7.20(1 \mathrm{H}, \mathrm{d}, J=7.22 \mathrm{~Hz}, \operatorname{Ar}-\mathrm{H}), 7.33(1 \mathrm{H}, \mathrm{t}, J=$ $8.08 \mathrm{~Hz}, \mathrm{Ar}-\mathrm{H}), 7.58(1 \mathrm{H}, \mathrm{d}, J=8.16 \mathrm{~Hz}, \mathrm{Ar}-\mathrm{H}), 7.86$ (s, Ar-H) 8.16 (1H, s, H-5), 9.50 (s, -NH-); ${ }^{13} \mathrm{C}$ NMR $\left(100 \mathrm{MHz}, \mathrm{CDCl}_{3}\right): \delta=118.16(\mathrm{Ur}-\mathrm{CH}), 118.36$, 120.28, 125.71, 130.29 (Ar-CH), 135.06, 137.53 (ArC), 157.96, 159.89, 160.02 (Ur-C), $165.41(\mathrm{C}=\mathrm{O})$. Anal. Calcd for $\mathrm{C}_{11} \mathrm{H}_{6} \mathrm{Cl}_{3} \mathrm{~N}_{3} \mathrm{O}: \mathrm{C}, 43.67 ; \mathrm{H}, 2.00 ; \mathrm{N}$, 13.89. Found: C, 43.90; H, 2.23; N, 13.75

2,4-dichloro-6-p-methylphenylamido pyrimidine (11). Off white crystal, yield: $5.53 \mathrm{~g}$ (83\%), mp 159$161{ }^{\circ} \mathrm{C}$; UV (EtOH): $\lambda_{\max } 320.00 \mathrm{~nm}$; IR (KBr): $v_{\max }$ 3369.4, 1687.6, 1566.1, 1525.6, 1317.3, 1294.1, $1245.9 \& 825.5 \mathrm{~cm}^{-1} ;{ }^{1} \mathrm{H}$ NMR (400 MHz, $\left.\mathrm{CDCl}_{3}\right): \delta$ $=2.34\left(3 \mathrm{H}, \mathrm{s}, \mathrm{Ar}-\mathrm{CH}_{3}\right), 7.18(2 \mathrm{H}, \mathrm{d}, J=8.28 \mathrm{~Hz}, \mathrm{Ar}-$ H), $7.59(2 \mathrm{H}, \mathrm{d}, J=8.32 \mathrm{~Hz}, \mathrm{Ar}-\mathrm{H}), 8.14(\mathrm{~s}, 1 \mathrm{H}, \mathrm{H}-$ 5), $9.43(\mathrm{~s},-\mathrm{NH}-) ;{ }^{13} \mathrm{C} \mathrm{NMR}\left(100 \mathrm{MHz}, \mathrm{CDCl}_{3}\right): \delta=$ $20.96\left(\mathrm{Ar}^{-} \mathrm{CH}_{3}\right), 118.22$ (Ur-CH), 120.06, 129.75 (Ar-CH),133.89, 135.39 (Ar-C), 157.60, 159.81, 160.46 (Ur-C), $165.13(\mathrm{C}=\mathrm{O})$. Anal. Calcd for $\mathrm{C}_{12} \mathrm{H}_{9} \mathrm{Cl}_{2} \mathrm{~N}_{3} \mathrm{O}: \mathrm{C}, 51.09 ; \mathrm{H}, 3.22 ; \mathrm{N}, 14.89$. Found: $\mathrm{C}$, $50.98 ; \mathrm{H}, 3.28 ; \mathrm{N}, 14.88$.

2, 4-Dichloro-6-m-methylphenylamido pyrimidine (12). Reddish crystal, yield: $5.46 \mathrm{~g}$ (82\%), mp 147-149 ${ }^{\circ} \mathrm{C}$; UV (EtOH): $\lambda_{\max } 356.00 \mathrm{~nm}$; IR $(\mathrm{KBr}): v_{\max } 3355.9,3068.7,1687.6,1531.4,1488.9$, 1309.6, 1296.1, $1247.9 \& 794.6 \mathrm{~cm}^{-1} ;{ }^{1} \mathrm{H}$ NMR $(400$ $\left.\mathrm{MHz}, \mathrm{CDCl}_{3}\right): \delta=2.41(3 \mathrm{H}, \mathrm{s}), 7.06(1 \mathrm{H}, \mathrm{d}, J=7.43$ $\mathrm{Hz}, \operatorname{Ar}-\mathrm{H}), 7.31(1 \mathrm{H}, \quad \mathrm{q}, J=7.61 \mathrm{~Hz}, \mathrm{Ar}-\mathrm{H}), 7.58$ $(2 \mathrm{H}, \mathrm{d}, J=9.42$, Ar-H) 8.18 (s, 1H, H-5), 9.46 (s, $\mathrm{NH}-) ;{ }^{13} \mathrm{C}$ NMR $\left(100 \mathrm{MHz}, \mathrm{CDCl}_{3}\right): \delta=21.49(\mathrm{Ar}-$ $\mathrm{CH}_{3}$ ), 117.28 (Ur-C), 118.29, 120.74, 126.46, 129.12 (Ar-CH), 136.34, 139.32 (Ar-C), 157.75, 159.91, 160.47 (Ur-C), $165.25(\mathrm{C}=\mathrm{O})$. Anal. Calcd for $\mathrm{C}_{12} \mathrm{H}_{9}$ $\mathrm{Cl}_{2} \mathrm{~N}_{3} \mathrm{O}: \mathrm{C}, 51.09 ; \mathrm{H}, 3.22 ; \mathrm{N}, 14.89$. Found: $\mathrm{C}$, 50.88; H, 3.26; N, 14.76 .

General procedure for the synthesis of 2,4dimethoxy-6-substituted phenylamido pyrimidines (13-17). 2, 4-Dichloro-6-substituted phenylamido pyrimidines (8-12) (1mmol) were added separately to the cold solution of sodium methoxide solution prepared by dissolving sodium $(3 \mathrm{mmol})$ in methanol $(30 \mathrm{ml})$. The mixture was refluxed at $60^{\circ} \mathrm{C}$ for 4 hours under Nitrogen atmosphere. After removal of solvent the crude mass was neutralized with dilute hydrochloric acid and extracted with chloroform $(3 \times 50 \mathrm{~mL})$. The combined chloroform layer was washed with distilled water $(2 \times 25 \mathrm{~mL})$, dried over anhyd $\mathrm{NaSO}_{4}$, filtered, and concentrated under reduced pressure. The residues were crystallized from methanol to obtain the desired products (13-17).

2,4-dimethoxy-6-p-methoxyphenylamido pyrimidine (13). Off white amorphous powder, yield: $3.34 \mathrm{~g}$ (82\%), mp 112-113 ${ }^{\circ} \mathrm{C}$; UV (EtOH): $\lambda_{\max }$ $286.00 \mathrm{~nm}$; IR (KBr): $v_{\max } 3332.8,3003.0,2947.0$, $1685.7,1589.2,1573.8,1535.2,1514.0,1483.2$, $1465.8, \quad 1384.8, \quad 1355.9, \quad 1315.4,1301.91263 .3$, $1232.4,1218.9,1201.6,1186.1,1186.1,1126.4$, 1076.2 1043.4, 987.5, $933.5 \& 769.5 \mathrm{~cm}^{-1} ;{ }^{1} \mathrm{H}$ NMR (400 MHz, $\left.\mathrm{CDCl}_{3}\right): \delta=3.79\left(3 \mathrm{H}, \mathrm{s}, \mathrm{Ar}-\mathrm{OCH}_{3}\right), 4.03$ $\left(3 \mathrm{H}, \mathrm{s}, \mathrm{Ur}-\mathrm{OCH}_{3}\right), 4.08\left(3 \mathrm{H}, \mathrm{s}, \mathrm{Ur}-\mathrm{OCH}_{3}\right) 6.90(1 \mathrm{H}$, d, $J=8.98 \mathrm{~Hz}, \mathrm{Ar}-\mathrm{H}), 7.25(1 \mathrm{H}, \mathrm{s}, \mathrm{H}-5), 7.64(1 \mathrm{H}, \mathrm{d}$, $J=8.98$, Ar-H) 9.54 (s, -NH-); ${ }^{13} \mathrm{C}$ NMR $(100 \mathrm{MHz}$, $\left.\mathrm{CDCl}_{3}\right): \delta=54.50\left(\mathrm{Ar}-\mathrm{CH}_{3}\right), 55.15,55.54(\mathrm{Ur}-$ $\left.\mathrm{OCH}_{3}\right), 100.39(\mathrm{Ur}-\mathrm{CH}), 130.38,133.09(\mathrm{Ar}-\mathrm{CH})$, 156.80, 159.22, 159.97 (Ur-C), $165.13(\mathrm{C}=\mathrm{O}))$. Anal. Calcd for $\mathrm{C}_{14} \mathrm{H}_{15} \mathrm{~N}_{3} \mathrm{O}_{4}$ : C, 58.13; H, 5.23; N, 14.53. Found: C, 58.00; H, 5.26; N, 14.76 .

2,4-dimethoxy-6-p-chlorophenylamido pyrimidine (14). White amorphous powder, yield: $3.39 \mathrm{~g}$ (86\%), mp 118-120 ${ }^{\circ} \mathrm{C}$; UV (EtOH): $\lambda_{\max } 282.00 \mathrm{~nm}$; IR (KBr): $v_{\max } 3355.9,3107.1,1691.5,1606.6$, $1583.4,1569.9,1519.8,1492.8,1460.0,1415.7$, $1398.3,1382.9,1303.8,1286.4,1238.2,1197.7$, 1093.6, 1024.1, 983.6, \& $829.3 \mathrm{~cm}^{-1} ;{ }^{1} \mathrm{H}$ NMR (400 $\left.\mathrm{MHz}, \mathrm{CDCl}_{3}\right): \delta=4.02\left(3 \mathrm{H}, \mathrm{s}, \mathrm{Ur}-\mathrm{OCH}_{3}\right), 4.06(3 \mathrm{H}$, $\left.\mathrm{s}, \mathrm{Ur}-\mathrm{OCH}_{3}\right), 7.22(1 \mathrm{H}, \mathrm{s}, \mathrm{H}-5), 7.31(2 \mathrm{H}, \mathrm{d}, J=8.78$ $\mathrm{Hz}, \mathrm{Ar}-\mathrm{H}), 7.65(2 \mathrm{H}, \mathrm{d}, J=8.8 \mathrm{~Hz}, \mathrm{Ar}-\mathrm{H}), 9.63$ (s, $\mathrm{NH}-) ;{ }^{13} \mathrm{C}$ NMR $\left(100 \mathrm{MHz}, \mathrm{CDCl}_{3}\right): \delta=54.56(\mathrm{Ur}-$ $\left.\mathrm{OCH}_{3}\right), 55.17\left(\mathrm{Ur}-\mathrm{OCH}_{3}\right), 100.50(\mathrm{Ur}-\mathrm{CH}), 118.83$, 119.89, 124.43, 126.57 (Ar-CH), 131.06, 131.22 (ArC), 145.29, 150.81, 151.81 (Ur-C), $164.03(\mathrm{C}=\mathrm{O})$. Anal. Calcd for $\mathrm{C}_{13} \mathrm{H}_{12} \mathrm{ClN}_{3} \mathrm{O}_{3}: \mathrm{C}, 53.16 ; \mathrm{H}, 4.12 ; \mathrm{N}$, 14.31. Found: C, 52.91; H, 4.11; N, 14.33.

2,4-dimethoxy-6-m-chlorophenylamido pyrimidine (15). Light pink amorphous powder, yield: $3.86 \mathrm{~g}$ (87\%), mp 120-121 ${ }^{\circ} \mathrm{C}$; UV (EtOH): $\lambda_{\max }$ 
$276.00 \mathrm{~nm} ; \mathrm{IR}(\mathrm{KBr}): v_{\max } 3355.9,3105.2,1706.9$, $1610.5,1598.9,1569.9,1508.2,1481.2,1475.4$, $1419.5,1396.4,1367.4,1290.3,1263.3,1201.6$, $1188.1 \& 1112.9 \mathrm{~cm}^{-1} ;{ }^{1} \mathrm{H}$ NMR $\left(400 \mathrm{MHz}, \mathrm{CDCl}_{3}\right)$ : $\delta=4.05\left(3 \mathrm{H}, \mathrm{s}, \mathrm{Ar}-\mathrm{OCH}_{3}\right), 4.09\left(3 \mathrm{H}, \mathrm{s}, \mathrm{Ar}-\mathrm{OCH}_{3}\right)$, $7.14(1 \mathrm{H}, \mathrm{d}, J=7.80 \mathrm{~Hz}$, Ar- $\mathrm{H}) 7.28(1 \mathrm{H}, \mathrm{dd}, J=$ 8.03, $7.78 \mathrm{~Hz}, \mathrm{Ar}-\mathrm{H}), 7.55(1 \mathrm{H}, \mathrm{d}, J=8.01 \mathrm{~Hz}, \mathrm{Ar}-$ H), 7.85 (1H, s, H-5), 9.66 (s, NH); ${ }^{13} \mathrm{C}$ NMR (100 $\left.\mathrm{MHz}, \mathrm{CDCl}_{3}\right): \delta=54.53\left(\mathrm{Ur}-\mathrm{OCH}_{3}\right), 55.17(\mathrm{Ur}-$ $\left.\mathrm{OCH}_{3}\right), 100.54$ (Ur-CH), 117.85, 119.96, 124. 83, 130.08 (Ar-CH), 134.79, 138.23 (Ar-C), 158.49, 160.33, 164.81 (Ur-C), 173.42 (-C=O). Anal. Calcd for $\mathrm{C}_{13} \mathrm{H}_{12} \mathrm{ClN}_{3} \mathrm{O}_{3}: \mathrm{C}, 53.16 ; \mathrm{H}, 4.12 ; \mathrm{N}, 14.31$. Found: C, 52.87; H, 4.01; N, 14.13 .

2,4-dimethoxy-6-p-methylphenylamido pyrimidine (16). Off white amorphous powder, yield: $3.29 \mathrm{~g}(85 \%), \mathrm{mp} 114-115^{\circ} \mathrm{C}$; UV (EtOH): $\lambda_{\max }$ $284.00 \mathrm{~nm}$; IR (KBr): $v_{\max } 3321.2,1676.0,1606.6$, $1585.4,1571.9,1525.6,1481.2,1458.1,1404.1$, 1390.6, $1361.7 \& 1049 \mathrm{~cm}^{-1}$; ${ }^{1} \mathrm{H}$ NMR $400 \mathrm{MHz}$, $\left.\mathrm{CDCl}_{3}\right): \delta=2.34\left(3 \mathrm{H}, \mathrm{s}, \mathrm{Ar}-\mathrm{CH}_{3}\right), 4.04(3 \mathrm{H}, \mathrm{s}, \mathrm{Ar}-$ $\left.\mathrm{OCH}_{3}\right), 4.09\left(3 \mathrm{H}, \mathrm{s}, \mathrm{Ar}-\mathrm{OCH}_{3}\right), 7.17(2 \mathrm{H}, \mathrm{d}, J=8.20$ $\mathrm{Hz}, \mathrm{Ar}-\mathrm{H}) 7.27(1 \mathrm{H}, \mathrm{s}, \mathrm{H}-5), 7.59$ (2H, d, $J=8.36$ $\mathrm{Hz}, \operatorname{Ar}-\mathrm{H}), 9.59$ (s, NH); ${ }^{13} \mathrm{C}$ NMR (100 MHz, $\left.\mathrm{CDCl}_{3}\right): \delta=20.92\left(\mathrm{Ar}^{-\mathrm{CH}_{3}}\right), 54.48\left(\mathrm{Ur}-\mathrm{OCH}_{3}\right), 55.13$ $\left(\mathrm{Ur}-\mathrm{OCH}_{3}\right), 100.42(\mathrm{Ur}-\mathrm{CH}), 119.89,129.62$ (Ar$\mathrm{CH}), 134.52,134.59$ (Ar-C) 159.14, 160.07, 164.79 (Ur-C), $173.43(-\mathrm{C}=\mathrm{O})$. Anal. Calcd for $\mathrm{C}_{14} \mathrm{H}_{15} \mathrm{~N}_{3} \mathrm{O}_{3}$ : C, 61.53; H, 5.53; N, 15.38. Found: C, 51.81; H, $5.41 ; \mathrm{N}, 15.33$.

2,4-dimethoxy-6-m-methylphenylamido pyrimidine (17). Off-white amorphous powder, yield: $3.37 \mathrm{~g}$ (87\%), mp 117-119 ${ }^{\circ} \mathrm{C}$; UV (EtOH): $\lambda_{\max }$ $302.00 \mathrm{~nm}$; IR (KBr): $v_{\max } 3319.1,1673.9,1609.6$, $1587.0,1571.9,1527.6,1495.2,1452.1,1404.1$, 1391.6, $1360.7 \& 1049 \mathrm{~cm}^{-1}$; ${ }^{1} \mathrm{H}$ NMR $(400 \mathrm{MHz}$, $\left.\mathrm{CDCl}_{3}\right): \delta=2.38\left(3 \mathrm{H}, \mathrm{s}, \mathrm{Ar}-\mathrm{CH}_{3}\right), 4.05(3 \mathrm{H}, \mathrm{s}, \mathrm{Ar}-$ $\left.\mathrm{OCH}_{3}\right), 4.10\left(3 \mathrm{H}, \mathrm{s}, \mathrm{Ar}-\mathrm{OCH}_{3}\right), 6.52(\mathrm{~m}, 1 \mathrm{H}, \mathrm{Ar}-\mathrm{H})$, $7.00(1 \mathrm{H}, \mathrm{d}, J=7.1 \mathrm{~Hz}, \mathrm{Ar}-\mathrm{H}) 7.27(\mathrm{~s}, 1 \mathrm{H}, \operatorname{Ar}-\mathrm{H})$, $7.28(1 \mathrm{H}, \mathrm{dd}, J=7.52 \mathrm{~Hz}, \operatorname{Ar}-\mathrm{H}), 7.52(1 \mathrm{H}, \mathrm{d}, J$ =7.99 Hz, Ar-H), 7.59 (1H, s, H-5), 9.66 (s, -NH-); ${ }^{13} \mathrm{C}$ NMR $\left(100 \mathrm{MHz}, \mathrm{CDCl}_{3}\right): \delta=21.47\left(\mathrm{Ar}-\mathrm{CH}_{3}\right)$, $54.48\left(\mathrm{Ur}-\mathrm{OCH}_{3}\right), 55.12\left(\mathrm{Ur}-\mathrm{OCH}_{3}\right), 100.45(\mathrm{Ur}-$ $\mathrm{CH}), 116.99,120.51,125.64,128.93,137.02,139.06$ (Ar-CH), 159.06, 160.15, 164.79 (Ur-C), 173.42 (-
$\mathrm{C}=\mathrm{O}$ ); Anal. Calcd for $\mathrm{C}_{14} \mathrm{H}_{15} \mathrm{~N}_{3} \mathrm{O}_{3}$ : C, 61.53; H, 5.53; N, 15.38. Found: C, 61.23; H, 5.62; N, 15.65 .

General procedure for the synthesis 6arylamido pyrimidine-2,4-diones (18-22). The demethylation reaction of 2,4-dimethoxy-6substituted phenylamido pyrimidines 13-17 were performed separately by refluxing with $6 \mathrm{M}$ hydrochloric acid aqueous solution for 4-6 hours. The reaction mixture was cooled and amorphous powder was separated by filtration, washed with chilled water, dried and crystallized from ethanol to afford 6substituted phenylamido uracils 18-22 in good yield.

6-p-methoxyphenylamido pyrimidine-2,4-dione

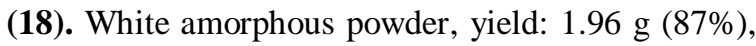
mp 276-277 ${ }^{\circ} \mathrm{C}$; UV (EtOH): $\lambda_{\max } 238.0 \mathrm{~nm}$; IR $(\mathrm{KBr}): \quad v_{\max } \quad 3296.1,3016.5, \quad 1735.8, \quad 1660.6$, 1618.2,1508.3, 1440.7, $837.0 \mathrm{~cm}^{-1}$; ${ }^{1} \mathrm{H}$ NMR (400 $\left.\mathrm{MHz}, \mathrm{DMSO}-d_{6}\right): \delta=3.74\left(3 \mathrm{H}, \mathrm{s}, \mathrm{Ar}-\mathrm{OCH}_{3}\right), 6.17$ $(1 \mathrm{H}, \mathrm{s}, \mathrm{H}-5), 6.95(2 \mathrm{H}, \mathrm{d}, J=8.96 \mathrm{~Hz}, \mathrm{Ar}-\mathrm{H}), 7.59$ $(2 \mathrm{H}, \mathrm{d}, J=8.96 \mathrm{~Hz}, \mathrm{Ar}-\mathrm{H}), 10.40(1 \mathrm{H}, \mathrm{s}, \mathrm{Ur}-\mathrm{NH})$, $10.87(1 \mathrm{H}, \mathrm{s}, \mathrm{Ur}-\mathrm{NH}), 11.29(\mathrm{~s}, 1 \mathrm{H},-\mathrm{NH}), ;{ }^{13} \mathrm{C} \mathrm{NMR}$ $\left(100 \mathrm{MHz}, \mathrm{DMSO}-d_{6}\right): \delta=55.21\left(\mathrm{Ar}-\mathrm{OCH}_{3}\right), 100.21$ (Ur-CH). 113.93, 121.98 (Ar-CH), 130.75, 145.67 (Ar-C), 150.79, 156.20, 158.39 (Ur-C), 164.09 ($\mathrm{C}=\mathrm{O})$; DEPT-135: $55.21\left(\mathrm{Ar}-\mathrm{OCH}_{3}\right), 100.21(\mathrm{U}-\mathrm{CH})$, 113.93, 121.98 (Ar-CH); Anal. Calcd for $\mathrm{C}_{12} \mathrm{H}_{11} \mathrm{~N}_{3} \mathrm{O}_{4}$ : C, 55.17; H, 4.24; N, 16.09. Found: $\mathrm{C}$, 55.00; H, 4.15; N, 16.10 .

\section{6-p-chlorophenylamidopyrimidine-2,4-dione}

(19). White amorphous powder, yield: $1.91 \mathrm{~g} \mathrm{(88 \% ),}$ mp 281-283 ${ }^{\circ} \mathrm{C}$; UV (EtOH): $\lambda_{\max } 356.00 \mathrm{~nm}$; IR $(\mathrm{KBr}): v_{\max } 3296.2,3095.5,2829.4,1735.8,1660.6$, 1596.9, 1529.4, 1498.6, 1440.7, $837.0 \mathrm{~cm}^{-1} ;{ }^{1} \mathrm{H}$ NMR (400 MHz, DMSO- $\left.d_{6}\right): \delta=6.18(\mathrm{~s}, 1 \mathrm{H}, \mathrm{H}-5), 7.44(\mathrm{~d}$, $1 \mathrm{H}, J=8.83 \mathrm{~Hz}, \mathrm{Ar}-\mathrm{H}), 7.71(\mathrm{~d}, 2 \mathrm{H}, J=8.85 \mathrm{~Hz}, \mathrm{Ar}-$ H), 10.64 (s, 1H, Ur-NH), 10.95 (s, 1H, Ur-NH), $11.32(\mathrm{~s}, 1 \mathrm{H},-\mathrm{NH}) ;{ }^{13} \mathrm{C}$ NMR (100 MHz, DMSO- $\left.d_{6}\right)$ : $\delta=100.71(\mathrm{Ur}-\mathrm{CH}), 118.83,119.89,124.43,130.57$ (Ar-CH), 133.06, 139.22 (Ar-C), 145.29, 150.81, 159.81 (Ur-C), $164.03(\mathrm{C}=\mathrm{O})$. Anal. Calcd for $\mathrm{C}_{11} \mathrm{H}_{8} \mathrm{ClN}_{3} \mathrm{O}_{3}: \mathrm{C}, 49.73 ; \mathrm{H}, 3.04 ; \mathrm{N}, 15.82$. Found: $\mathrm{C}$, 49.92; H, 3.28; N, 15.53 .

\section{6-m-chlorophenylamidopyrimidine-2,4-dione}

(20). White amorphous powder, yield: $1.69 \mathrm{~g} \mathrm{(85 \% ),}$ 
mp 274-275 ${ }^{\circ} \mathrm{C}$; UV (EtOH): $\lambda_{\max } 350.00 \mathrm{~nm}$; IR $(\mathrm{KBr}): v_{\max } 3157.3,2922.0,1721.7,1591.2,1542.9$, 1458.1, $1016.4 \mathrm{~cm}^{-1}$; ${ }^{1} \mathrm{H}$ NMR (400 MHz, DMSO$\left.d_{6}\right): \delta=6.17(1 \mathrm{H}, \mathrm{s}, \mathrm{H}-5), 7.23(1 \mathrm{H}, \mathrm{d}, J=7.89 \mathrm{~Hz}$, Ar-H), 7.41(1H, t, $J=8.1 \mathrm{~Hz}, \mathrm{Ar}-\mathrm{H}), 7.61(1 \mathrm{H}, \mathrm{d}, J=$ $8.37 \mathrm{~Hz}, \mathrm{Ar}-\mathrm{H}), 7.85$ (1H, s, Ar-H), 10.67 (1H, s, Ur$\mathrm{NH}), 10.97(1 \mathrm{H}, \mathrm{s}, \mathrm{Ur}-\mathrm{NH}), 11.34(\mathrm{~s},-\mathrm{NH}) ;{ }^{13} \mathrm{C}$ NMR (400 MHz, DMSO- $\left.d_{6}\right): \delta=100.71(\mathrm{Ur}-\mathrm{CH})$, 118.83, 119.89, 124.43, 130.57 Ar-CH), 133.06, 139.22 (Ar-C), 145.29, 150.81, 159.19 (Ur-C), $164.03(\mathrm{C}=\mathrm{O})$. DEPT-135: $\delta_{\mathrm{C}} 100.71 \quad(\mathrm{Ur}-\mathrm{CH})$, $118.82,119.82,124.43,130.56$ (Ar-CH).

6-p-methylphenylamido pyrimidine-2,4-dione

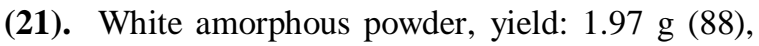
mp 267-269 ${ }^{\circ} \mathrm{C}$; UV (EtOH): $\lambda_{\max } 278.00 \mathrm{~nm}$; IR $(\mathrm{KBr}): v_{\max } 3280.5,3014.5,1735.4,1616.2,1505.8$, 1488.4, 1265.6, $1110.6 \& 802.3 \mathrm{~cm}^{-1} ;{ }^{1} \mathrm{H}$ NMR (400 $\left.\mathrm{MHz}, \mathrm{DMSO}-d_{6}\right): \delta=2.30\left(3 \mathrm{H}, \mathrm{s}, \mathrm{Ar}-\mathrm{CH}_{3}\right), 5.67$ $(1 \mathrm{H}, \mathrm{s}, \mathrm{H}-5), 6.68(2 \mathrm{H}, \mathrm{d}, J=8.18 \mathrm{~Hz}, \mathrm{Ar}-\mathrm{H}), 7.06$ (d, $2 \mathrm{H}, J=8.20 \mathrm{~Hz}, \mathrm{Ar}-\mathrm{H}), 9.94(1 \mathrm{H}, \mathrm{s}, \mathrm{Ur}-\mathrm{NH}), 10.39$ $(1 \mathrm{H}, \mathrm{s}, \mathrm{Ur}-\mathrm{NH}), 10.80(1 \mathrm{H}, \mathrm{s},-\mathrm{NH}) ;{ }^{13} \mathrm{C}$ NMR $(100$ MHz,DMSO- $\left.d_{6}\right): \delta=20.49\left(\mathrm{Ar}-\mathrm{CH}_{3}\right), 100.33(\mathrm{Ur}-$ $\mathrm{CH}), 120.41,129.19(\mathrm{Ar}-\mathrm{CH}), 133.82,135.24(\mathrm{Ar}-$ C), 145.66, 150.81, 158.65 (Ur-C), 164.10 (-C=O). DEPT-135: $\delta_{\mathrm{C}} 20.49\left(\mathrm{Ar}-\mathrm{CH}_{3}\right), 100.33(\mathrm{Ur}-\mathrm{CH})$, 120.41, 129.19(Ar-CH); Anal. Calcd for $\mathrm{C}_{12} \mathrm{H}_{11} \mathrm{~N}_{3} \mathrm{O}_{3}$ : $\mathrm{C}, 58.77 ; \mathrm{H}, 4.52 ; \mathrm{N}, 17.13$. Found: $\mathrm{C}, 58.60 ; \mathrm{H}$, $4.68 ; \mathrm{N}, 16.87$.

6-m-methylphenylamido pyrimidine-2,4-dione (22). White amorphous powder, yield: $1.90 \mathrm{~g}(85 \%)$, mp 262-264 ${ }^{\circ} \mathrm{C}$; UV (EtOH): $\lambda_{\max } 242.00 \mathrm{~nm}$; $\operatorname{IR}(\mathrm{KBr}): v_{\max } 3157.3,2925.8, \quad 1712.7,1614.3$, $1560.3,1488.8, \quad 1369.5,1266.2,1113.8,1020.2$ $\mathrm{cm}^{-1}$; ${ }^{1} \mathrm{H}$ NMR (400 MHz, DMSO- $\left.d_{6}\right): \delta=2.30(\mathrm{~s}$, $3 \mathrm{H}, \mathrm{Ar}-\mathrm{CH}_{3}$ ), 6.17 (s, 1H, H-5), 6.98 (d, 1H, $J=7.5$ $\mathrm{Hz}, \mathrm{Ar}-\mathrm{H}), 7.25((1 \mathrm{H}, \mathrm{t}, J=7.83 \mathrm{~Hz}, \mathrm{Ar}-\mathrm{H}), 7.47$ $(1 \mathrm{H}, \mathrm{d}, J=8.31 \mathrm{~Hz}, \mathrm{Ar}-\mathrm{H}), 7.52(1 \mathrm{H}, \mathrm{s}, \mathrm{Ar}-\mathrm{H}), 10.44$ (1H, s, Ur-NH), 10.91 (1H, s, Ur-NH), 11.31 (1H, s, $\mathrm{NH}) ;{ }^{13} \mathrm{C}$ NMR $\left(100 \mathrm{MHz}, \mathrm{DMSO}-d_{6}\right): \delta=21.14$ $\left(\mathrm{Ar}-\mathrm{CH}_{3}\right), 100.41$ (Ur-CH), 117.61, 120.92,125.29, 128,65 (Ar-CH), 137, 138.06 (Ar-C), 145.62, 150.84, $158.81(\mathrm{Ur}-\mathrm{C}), 164.11(\mathrm{C}=\mathrm{O})$.

\section{RESULTS AND DISCUSSION}

It has been reported a very facile method for the synthesis of a number of 6-amidopyrimidine-2,4diones from orotic acid. 2,4- Dichloropyrimidine-6carbonyl chloride (2) was synthesized according to the procedure of Gershon ${ }^{10}$ by heating Orotic acid $\mathbf{1}$ with phosphorus oxychloride and phosphorus pentachloride as shown in the scheme 1. Phosphorus oxychloride was recovered under reduced pressure. The residue was distilled under reduced pressure and 2,4-dichloropyrimidine-6-carbonyl chloride (2) (4.0 g) was obtained as dense colorless liquid.

The compound 2,4-dichloropyrimidine-6carbonyl chloride $\mathbf{2}$ underwent a smooth reaction with a number of substituted amine derivatives in which the acid chloride moiety was found to react predominantly to produce desired product 2,4dichloro-6-substituted phenylamido pyrimidines (812) as shown in the Scheme-1.

2,4-dichloro-6-substitutedphenylamidopyrimidines (8-12) were converted to the corresponding dimethoxy pyrimidines (13-17) on treatment with sodium methoxide in methanol as shown in the scheme 1 and Table 1. After usual workup the residues were crystallized from methanol and 2,4dimethoxy-6-substituted phenylamidopyrimidines (13-17) were obtained in good yield (82-87\%). The demethylation reaction of 2,4-dimethoxy-6substituted phenylamido pyrimidines (13-17) were performed by heating with $6 \mathrm{M}$ hydrochloric acid aqueous solution for 4-6 hours and 6-substituted phenylamido uracils (18-22) were obtained in good yield (85-88\%) after usual workup as shown in the scheme 1 and Table 1 . The compounds (18-22) were crystallized from ethanol.

In conclusion, a convenient and facile method is developed for the synthesis of 6-amido pyrimidine-2, 4-diones from orotic (uracil-6-carboxylic acid) acid. The purification of the synthesized compounds were very simple, mainly crystallization. A variety of functional groups can be introduced at the C-6 positions of the pyrimidine ring by this procedure. It is believed that the synthesized pyrimidinone derivatives might show antiviral activity as reported in literature. We are planning to test our compound against virus. 

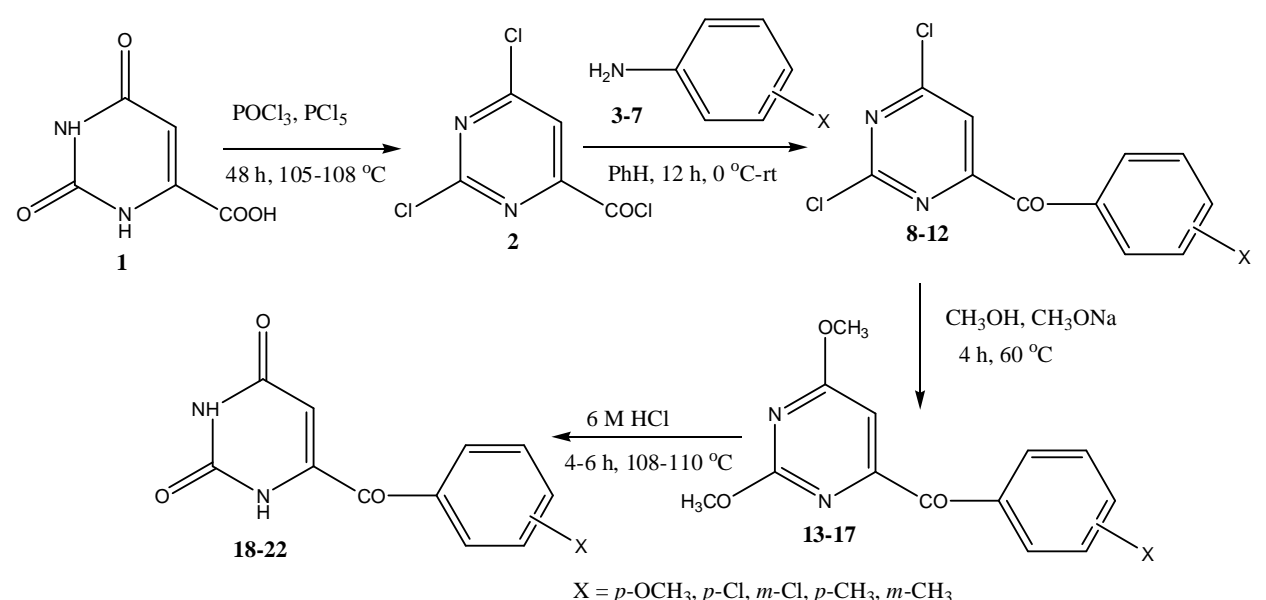

Scheme 1

Table 1. Synthesis of 2,4-dichloro-6-substituted phenylamidopyrimidines (8-12); 2,4-dimethoxy-6-arylamido pyrimidines (13-17); 6-arylamido pyrimidine-2, 4-diones (18-22)

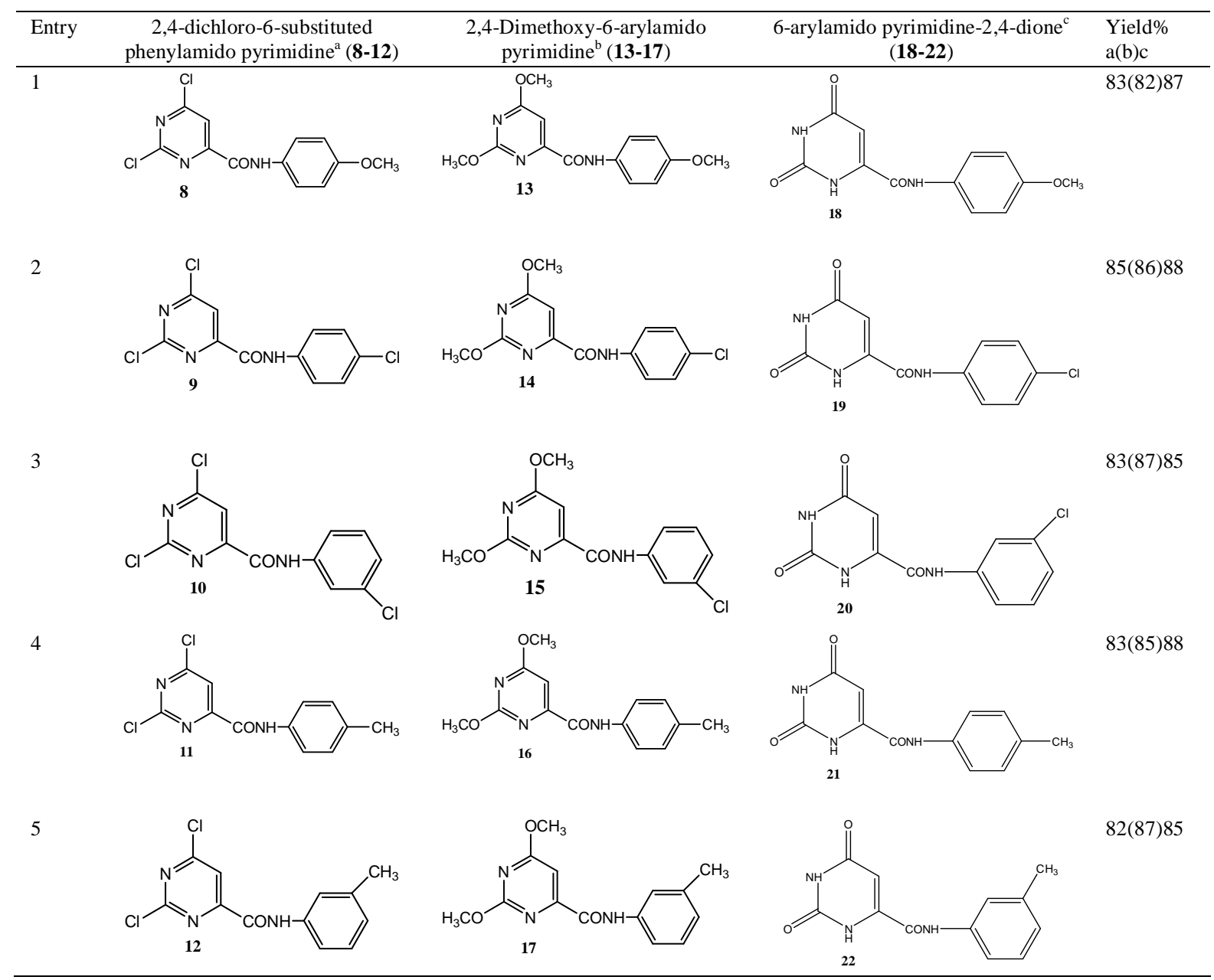

${ }^{\mathrm{a}}$ Yield\% based on 2,4-dichloropyrimidine-6-carbonyl chloride 2; ${ }^{\text {b } Y i e l d \% ~ b a s e d ~ o n ~ 2,4-d i c h l o r o-6-s u b s t i t u t e d ~ p h e n y l a m i d o ~ p y r i m i d i n e s ~}$ (8-12); ${ }^{\mathrm{c}}$ Yield\% based on 2,4-dimethoxy-6-substituted arylamido pyrimidines (13-17) 


\section{ACKNOWLEDGEMENTS}

Financial support from Bangladesh University of Engineering and Technology, Dhaka, Bangladesh is gratefully acknowledged. We are grateful to Prof. Koichi Kato, Nagoya City University, Nagoya, Japan for taking CHN analyses. Thanks are also due to Prof. Kazuaki Shimada, Iwate University, Morioka, Japan for taking NMR spectra.

\section{REFERENCES}

1. Heiedelberger, C. 1984. Pyrimidine and pyrimidine nucleoside antimetabolites, in cancer medicine, Eds. Holland, J. F., and Frei, E., Lea and Febiger, Philadelphia, pp. 801.

2. Prusoff, W.H. and Fisher, P.H. In Nucleoside Analogues: Chemistry, biology and medical applications, Eds. Walker, R. T., DeClerq, E., Eckstein, F., vol. 26A, Plenum press, New York, N.Y., pp. 281-318.

3. Artico, M., Massa, S., Mai, A., Marongiu, M.E., Piras, G., Tramontano, E. and La colla, P. 1993. 3, 4-Dihydro-2alkoxy-6-benzyl-4-oxopyrimidinees (DABOs): A new class of specific inhibitors of human immunodeficiency vVirus Type 1. Antiviral Chem. Chemther. 4, 361-368.

4. Tramontano, E., Marongiu, M.E., De Montis, A., Loi, A.G., Artico, M., Massa, S., Mai, A. and La Colla, P. 1994. Characterization of the Anti-HIV-1 Activity of 3, 4-Dihydro2-alkoxy-6-benzyl-4-oxopyrimidines (DABOs), New Non- nucleoside Reverse Transcriptase Inhibitors. Microbiologica 17, 269-279.

5. Massa, S., Mai, A., Artico, M., Shardella, G., Tramontano, E., Loi, A. G., Scano, P. and La Colla, P. 1995. Synthesis and antiviral activity of new 3,4-dihydro-2-alkoxy-6-benzyl-4oxopyrimidines (DABOs), specific inhibitors of human immunodeficiency virus type 1. Antiviral Chem. Chemother. 6, 1-8.

6. Mai, A., Artico, M., Shardella, G., Massa, S., Loi, A.G., Tramontano, E., Scano, P. and Colla, P.L. 1995. Synthesis and anti-HIV-1 activity of thio analogues of dihydroalkoxybenyloxopyrimidines. J. Med. Chem. 39, 32583263.

7. Miyasaka, T., Tanaka, H., Baba, M., Haykawa, H., Walkr, R. T., Balzarini, J. and De Clercq, E. 1989. A novel lead for specific anti-HIV-1 agents: 1-[(2-hydroxyethoxy)methyl]-6(phenylthio)thymine. J. Med. Chem. 32, 2507-2509.

8. Brollosy, El., Jorgensen, P.T., Dahan, B., Boel, A.M., Pedersen, E.B. and Nielsen, C. 2002. Synthesis of novel N-1 (allyloxymethyl) analogues of 6-benzyl-1-(ethoxymethyl)-5isopropyluracil (MKC-442, emivirine) with improved activity against HIV-1 and its mutants. J. Med. Chem. 45, 5721-5726.

9. Khan, M.W. and Kundu, N.G. 1999. An expeditious synthesis of 4-acyl-2, 6-dioxo-1, 2, 3, 6-tetrahydropyrimidines (6-acyl uracils) and 4-acyl-6-aryl-2-oxo-2, 3dihydropyrimidines. J. Chem. Research(s) 20-21, 0301-0318.

10. Gershon, H. 1962. Chlorinated pyrimidines derived from orotic acid. J. Org. Chem. 27, 3507-3510. 